\title{
An Adaptable Full-Scale Aircraft Cabin in an Interconnected Simulation Environment
}

\author{
Mario Kallenbach, Stephan Kocks, Paul Frost, Ingo Voissel, Peter Hecker \\ Institute of Flight Guidance, Technische Universität Braunschweig, Hermann-Blenk-Str. 27, \\ 38108 Braunschweig, Germany; m.kallenbach|s.kocks|p.frost|i.voissel|p.hecker@tu-braunschweig.de
}

SNE 29(1), 2019, 27-32, DOI: 10.11128/sne.29.tn.10463

Received: March 18,2019 (Selected ASIM GMMS/STS 2019

Conference Publication); Accepted; March 12, 2019

SNE - Simulation Notes Europe, ARGESIM Publisher Vienna, ISSN Print 2305-9974, Online 2306-0271, www.sne-journal.org

Abstract. An aircraft cabin is - apart from interactions with the cabin crew - the most important interface between an airline and its passengers. The Institute of Flight Guidance (IFF) of the Technische Universität Braunschweig developed and built a full-scale aircraft cabin section comprising the structure and electronic systems of a short- to medium-haul single-aisle commercial aircraft. In this paper, we describe the ongoing work of setting up the cabin simulation and the developed solutions to a variety of technical challenges. We present our structural hard- and software architecture focusing on high realism or plausibility while maintaining a high flexibility in the design for future developments. By means of two more detailed examples - an outside view simulation system and an interconnection network for the simulation environment - we show our approach.

\section{Introduction}

The most lasting impressions (positive or negative) of a past flight come from aspects related to the aircraft cabin interior. Apart from pre-flight experiences and crew service quality, studies (e.g., [1] [2]) show that cabin systems have a high impact on the revenue of an airline through passenger comfort and the resulting desire to re-book with the same airline and/or the same aircraft. Although the cabin is partly subject to high safety requirements, its influence on the safe execution of the flight is mainly secondary. Thus, it offers more chances for the successful introduction of new developments than other parts of the aircraft structure. These aspects are contributing to the fact that aircraft cabin systems increasingly receive attention both in research and economic contexts.

New procedures and systems aim at the sometimes contradicting aspects of increased safety, greater operational efficiency and an improvement in passenger comfort. For their validation, models and simulations are required since a test during real-life operation is often too time- and cost-intensive. However, the multitude of cabin systems and the necessity of simulating a large number of influences on the operation in the cabin, pose a variety of challenges for the technical simulation.

In order to meet the resulting requirements of educational and research purposes, the Institute of Flight Guidance (IFF) of the Technische Universität Braunschweig developed and built a full-scale aircraft cabin section of a short- to medium-haul single-aisle commercial aircraft. The fixed-base cabin section is attached to an aircraft cockpit simulator and embedded into a superordinate simulation environment.

In this paper we describe the ongoing work of setting up the cabin simulation and the developed solutions to the mentioned technical challenges. We present our structural hard- and software architecture focusing on high realism or plausibility while maintaining a high flexibility in the design for future developments.

As a detailed example, we show the currently implemented outside view simulation and discuss the technical and perception-based challenges faced during development. Furthermore, the results of an examination with multiple test persons are featured.

Our subsequent outlook on future work contains ideas on an approach for data exchange. We envisage a publish-subscribe-based architecture to not only connect the individual components within the cabin simulator, but to also enable the interaction with other participants within the surrounding simulation environment as well as in external networks. 


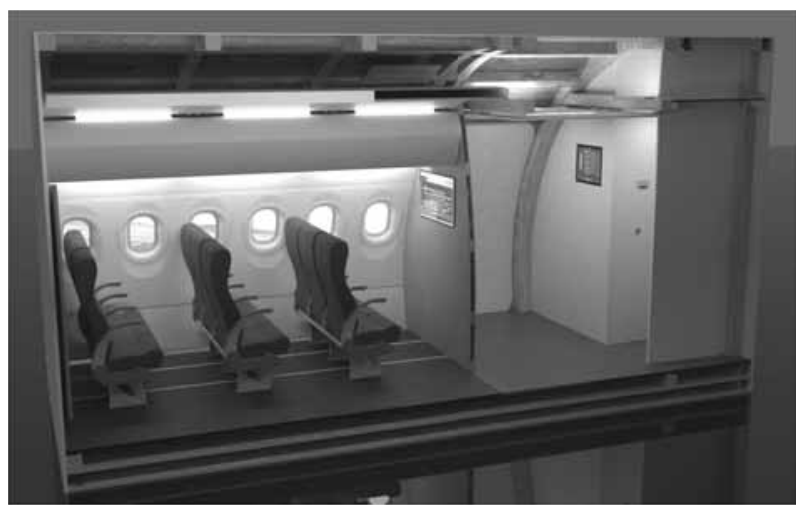

Figure 1: Computer-rendered representation of the cabin simulator cross-section.

\section{Simulator Architecture}

The fixed-base aircraft cabin simulator comprises the structure and electronic systems of the entrance area including the forward galley and lavatory as well as the full lining of the the first three to four seat rows in the passenger cabin deck (cf. Figure 1). It is equipped with relevant cabin systems and can be flexibly adapted to test systems from current research. The focus is on networking, sensor technology and human-in-the-loop simulations for the optimization of cabin-related processes. The cabin simulator is spatially adjacent and electronically connected to the aircraft cockpit simulator of the IFF and therefore constitutes part of a fullscale airliner simulation platform, which is orientated on an Airbus A320. This platform in turn is interconnected to other simulation systems and embedded into an air traffic management simulation environment consisting of a Diamond DA42 simulator and two working positions, each of which can be operated either by a (pseudo) pilot or an air traffic controller. This enables us to not only investigate the processes and interactions between cockpit and cabin, but also to consider the cabin as a further entity within the overall air traffic transport system.

\subsection{Structure}

The integration and evaluation of new and further developments requires a continuous adaptation of the cabin structure. For this reason, a modular approach was chosen, which makes it possible to flexibly modify or replace cabin modules, parts and systems. Furthermore, in order to give test persons a realistic impression, great importance was attached to reproducing the look and feel of the interior as true to original as possible and in particular the inner dimensions. The outer dimensions of the cabin are slightly larger in order to provide more space behind the lining components.

The basic structure of the cabin simulator is fully composed of wooden ribs and beams in combination with wooden panels and walls. Due to a reinforced floor structure, the cabin simulator can be easily lifted and moved. The outer shell is made of large PVC sheets. The cut-outs of the cabin doors on both sides are already in place, while the actual doors have already been designed, but not yet manufactured. In total, the cabin is approximately $5.6 \mathrm{~m}$ long, $4.2 \mathrm{~m}$ wide and $3.0 \mathrm{~m}$ in height and features twelve cabin windows (six on either side), which however do not have an opening to the outside.

For the lining of the passenger area, mostly used but refurbished original parts - such as window panels, ceiling panels and overhead bins - were incorporated. The total of 18 passenger seats (three groups of three seats on either side) are used originals as well and have a fold-out cocktail table to establish a business configuration. All other interior parts (e.g. the floor carpet) have been selected or replicated to resemble their counterpart in reality. Two self-developed bulkheads separate the passenger area from the entrance area. Except for the forward galley on the right-hand side, the entrance area was reconstructed for the most part. The lavatory on the left-hand side in the passage to the flight deck is only a dummy. It contains the computers and most of the other hardware components for controlling the cabin simulator as described in the following section.

\subsection{Hardware}

The cabin hardware offers input and output devices as human-machine interface for cabin operation on the one hand and on the other hand provides computing power for the simulated systems on board as well as for the environment simulation. In addition, it provides the basis for stimulating human senses through the visual and auditive channel; the haptic stimulus results from the structure and interior. The cabin simulator has its own power grid, which is divided into sub-grids by a main distributor. For cabin operation, five computers are used that are connected via Ethernet: two high-performance computers, each with two graphics cards for the outside view simulation and ambient noise as well as one computer each for internal audio and video streaming, 
for the Cabin Management System (CMS) and Flight Attendant Panel (FAP) and for the provision of various interfaces and aircraft buses (e.g. ARINC 429, RS232, RS485, CAN, etc.).

For the illumination of the cabin simulator, pairs of bright white and RGB LED strips were installed that are controlled independently via Digital Multiplex (DMX) dimmers. Within the passenger area, the LED strips are located on both sides of the ceiling panels, below the overhead bins and on floor level; within the entrance area, multiple pairs of LED strips are mounted in the ceiling only.

The output of cabin internal audio signals takes place via structure-borne sound speakers mounted on the ceiling panels. A separated surround system hidden behind the lining is used for the aircraft and ambient sounds. To enable the outside-view simulation (see Section 2), one screen is mounted behind each of the twelve cabin windows, three of which are connected via daisy chain to one graphics card of the highperformance computers. A large-scale screen is also installed in each of the two bulkheads for information and In-Flight Entertainment (IFE). Additionally, a touchscreen is available attached to the lavatory wall within the entrance area. The installation of a ventilation system for the air conditioning of the cabin is currently in progress. While the air conditioning unit and the ventilation outlets are already installed, the individual components are not yet connected.

\subsection{Software}

Building on the hardware and structural features, the systems and functions of the simulator are represented in a set of programs and software parts. These are classified into mock-ups, device simulation modules and auxiliary modules. Mock-ups mimic real software which is available in a real operational context. An example of a mock-up software is the FAP software implemented for the simulator. Device simulation modules imitate the behavior as well as the usage of cabin hard- or software that however is not physically available in the simulator. Most times they replace real hardware - e.g. lavatory equipment. Auxiliary modules, on the contrary, do not match any real world software or device. They are necessary to provide interfaces between virtual and real hardware, provide functions for the simulation control and serve as abstraction layer between cabin systems and simulator systems.
While the mock-up software and the auxiliary modules are implemented as stand-alone binaries, interconnected via TCP/IP-based interprocess-communication, device simulation modules heavily depend on a simulation framework we named Cabin Simulation Environment (CaSE). CaSE provides such features as eventbased data exchange, scenario generation and replay as well as lock-free concurrent interfaces to a variety of data buses. This ensures that new simulation devices can be implemented as light-weight components enabling developers to focus on remodeling the real device's behavior and providing seamless integration.

Device simulation modules may both simulate the behavior of a device and the usage of cabin systems by passengers and crew. Respective scenarios, triggering actions of virtual humans in the cabin and in turn leading to reactions by device simulation modules, can be generated and replayed using the graphical user interface of CaSE. For example, modules are available for the usage of lavatories (door locks, call buttons, water tank levels) or temperature control. Thus, as shown in Figure 2, CaSE expands the simulation from the first three seating rows to a full aircraft cabin layout by providing virtual additions to the real hardware.



Figure 2: Schematic representation of the cabin simulator comprising the real and simulated hardware of the cabin interior, cabin systems and their usage.

Central component of the whole software architecture is the mock-up software for the CMS. It provides information for and communication between systems and takes control of all connected systems in the cabin. The interface between this management system and the flight crew is the FAP. Its main purpose is to provide a graphical user interface for interacting with all systems on board and to be the link to the CMS. The FAP software is visible at the touch-screen monitor in the front section of the cabin (cf. Figure 1), which is also the corresponding input device. 
The auxiliary modules, generally also connected via the CMS, serve as interfaces and converters to the their respective connected hardware. For example, the lighting module provides pre-defined mood lighting scenarios which can be selected at the FAP. The resulting request is sent via the CMS to the lighting module, where it is converted into an ARTNet-compliant message which triggers the DMX dimmer hardware. Another auxiliary module serves as media streaming device for the IFE and passenger announcement system. Thus, it grants access to the bulkhead monitors and the structure-borne sound speakers (cf. Section 1.2) for video and audio transmission into the cabin.

\section{Outside-View Simulation}

The visual perception is one of the key senses to be stimulated for a high immersion of the participants of studies. This does not only apply to the interior, where materials, forms and colors have to match the past experience of the passengers. Instead, it applies especially to the view of the outside world which is provided through the cabin windows. Because of the fixed-base character of the simulator, this is the main way to create the impression of motion of the simulated aircraft. On the other hand, errors and misconceptions of this simulation component may easily lead to discomfort or even Simulation Sickness Syndrome (SSS).

Our 12-monitor-setup and twelve independently generated views provide a realistic outside view for test persons. Apart from bandwidth concerns and image generation performance, the selection of the depicted content and the implementation of optically correct perspectives were addressed while developing the system. The main challenge the hardware setup poses is the contradiction between multiple persons looking from different positions and directions to the same fixed, 2D representation of a 3D outside view. However, we expect that deviations from reality are easily accepted if a plausible view is obtained that matches the test person's past experience. We targeted at exploiting this feature by averaging the views to minimize the perceptible deviations between the simulation and reality. We used human-in-the-loop simulations with multiple test persons to evaluate the level of immersion as well as the impact on the involved humans with respect to visual perception of movement while lacking a kinesthetic stimulus.

\subsection{Architecture and setup}

The system uses the hardware architecture described in Section 1.2 with twelve screens in the outer walls and can therefore access a total of two CPUs and four GPUs for image generation. The used software strongly influences the available visual content, performance and synchronizing features. Thus, the experiments were conducted using varying software platforms, more specifically, Laminar Research's X-Plane 11, Prepar3D by Lockheed Martin and - deviant from using a flight simulator software - Google Earth Pro.

The two flight simulation programs were set up to have one master instance, calculating the flight status vector using the flight model and three to five slave instances which only generated synchronized views with different viewing angles of the environment. Both flight simulations provide configuration files for setting up the correct parameters of the outside view. In case of Prepar3D, multiple views can be specified together. For $\mathrm{X}$-Plane, different instances of the software have to be launched. However, this also allows for the separate instances to use different associated GPUs, balancing the load of image generation across the available hardware. Another challenge is synchronicity and temporal resolution, which is not only crucial for holding up the illusion of steady movement. When using different software instances, information like the shape and position of clouds, ground vehicles and other traffic must be interchanged or propagated. Both simulations use TCP/IP-based proprietary protocols for that purpose.

Google Earth Pro instead was connected to the flight simulation of the adjacent cockpit simulator used as data provider and is only capable of one sole view which was stretched across the screens. Though we tested this setup, we did not consider it for the evaluation as the huge drawbacks of this approach stood out at an early stage.

\subsection{Viewing layouts}

In order to consolidate and average the multitude of possible viewing directions and positions to a set of views that can be shown on the different screens, we considered different combinations of number of viewing points, number of independent views of these points and positions of the viewing points. These layouts, of which three were later used for the evaluation, are defined in Table 1. 


\begin{tabular}{l|l|l|l}
\hline & $\begin{array}{l}\text { Viewing Points } \\
\text { (VP), in total }\end{array}$ & $\begin{array}{l}\text { Views } \\
\text { per VP }\end{array}$ & VP Height \\
\hline Row layout & 6 & 2 & $1175 \mathrm{~mm}$ \\
\hline Aisle layout (high) & 1 & 12 & $1570 \mathrm{~mm}$ \\
\hline Aisle layout (low) & 1 & 12 & $1033 \mathrm{~mm}$ \\
\hline Duplicated layout & 1 & 2 & $1175 \mathrm{~mm}$ \\
\hline Single layout & 6 & 2 & $1175 \mathrm{~mm}$ \\
\hline
\end{tabular}

Table 1: Configurations of view layouts.

Each layout entails advantages and disadvantages concerning feature visibility, degree of realism, generation performance, etc., depending on the passengers position in the cabin and the simulated flight phase. Although the according considerations were made, the detailed discussion exceeds the scope of this paper.

In Figure 3, the different viewing layouts resulting from varying the input parameters are schematically depicted. The chosen layouts determine the optical parameters of the system, which in turn can be calculated and implemented in the used software.

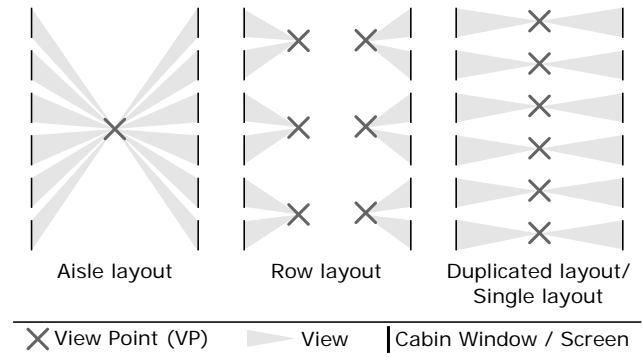

Figure 3: Schematic depiction of view layouts in the cabin simulator (top view).

\subsection{Evaluation}

For the evaluation of our solution, we conducted human-in-the-loop tests including four different scenarios, each with a duration of approximately $15 \mathrm{~min}-$ utes. Each scenario contained a short taxi phase, takeoff phase and a climb phase up to a virtual altitude of $12000 \mathrm{ft}$. While climbing, the aircraft performed both a left and a right turn. In the different scenarios, the viewing layout and the software platform were varied, see Table 2 for reference. The 34 test persons were asked to choose a seat and fill in a questionnaire asking for grades from 1 to 10 concerning the assessment of realism, image quality, temporal resolution, synchronic-

\begin{tabular}{l|l|l}
\hline & Viewing layout & Software \\
\hline Scenario 1 & Row layout & X-Plane \\
\hline Scenario 2 & Aisle layout (high) & X-Plane \\
\hline Scenario 3 & Aisle layout (low) & X-Plane \\
\hline Scenario 4 & Aisle layout (low) & Prepar3D \\
\hline
\end{tabular}

Table 2: Input parameters for the evaluation scenarios.

ity, impression of movement and personal well-being taking the flight phase into account. Also, test persons were asked to look at the outside view from different positions in the cabin, at least once from their seat and once from the aisle. Combined with (optional) test person specific information concerning age, gender, body height as well as frequency of flight, this produces comprehensive data for the evaluation of the implemented system. As the excerpt from the results in

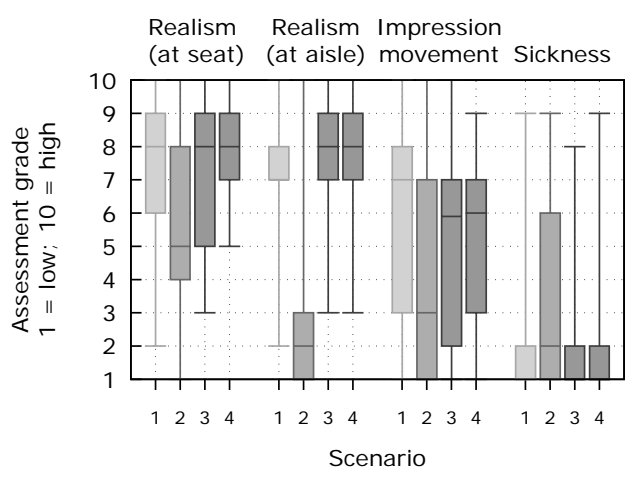

Figure 4: Grades concerning assessment of realism (while viewing from seat or aisle), of impression of movement and of individual sickness.

Figure 4 indicates, the system generally provides a good depiction of reality. The assessment concerning the impression of movement shows potential for improvement as the grades are on an average level. This feedback was to be expected since the simulator does not provide kinesthetic stimuli in the form of perceptible accelerations. Despite this missing sensation, which contradicts the test persons' observations, the experienced sickness level is low except for scenario 2. In general, scenario 2 shows the worst performance, especially when the simulation is viewed from the aisle. Test persons criticized the high visual tilt downwards in that scenario.

The overall results from the extensive study substantiated our tendency towards scenario 3. Thus, we selected this scenario for operation. 


\section{Interconnection Network}

The interconnection of the simulators described in Section 1 should enable a composite simulation. Each simulator will be considered as a single entity within the simulation structure. Nevertheless, the cabin and cockpit simulators constitute a single entity in most of the use cases. Currently, each entity operates within its own network and - if required - the intercommunication is fulfilled via dedicated interfaces.

\subsection{Requirements}

The aim of the next stage of development is to provide a more flexible connection between the entities and other modules. Therefore, it shall be possible to operate each entity individually and as a part of a composite simulation. For a quick integration of external modules, the interfaces have to be easily accessible. However, the interfaces must keep step with the development of systems.

\subsection{Envisaged implementation}

In order to meet the requirements and to avoid the occurrence of single point of failure events, it is foreseen to use a distributed system design such as the Robot Operating System 2 (ROS 2) to connect the simulators. ROS 2 uses Fast Real Time Publish-Subscribe (RTPS) [3] which builds on the Data Distribution Service (DDS) middleware.

Using ROS 2, systems are able to publish data within self-defined topics to provide them to other entities. Participants can subscribe to the topics they require. On the application layer there is no further communication necessary between the entities and there is no central instance required for controlling the communication flow. Discovery of the participants is also handled transparently by the middleware.

Each topic holds one data type. Custom data structures have to be defined in Interface Definition Language (IDL) files provided for every system at compile time. It is envisaged to only use primitive data types as well as their corresponding arrays at first. Therefore, most of the data fields provided by our simulators are to be transferred within individual topics. Complex data types will be defined subsequently, if needed.

Since Fast RTPS internally uses UDP/TCP as transport protocol, it is mandatory that the subnets of each simulation entity will be connected within the Local
Area Network (cf. Figure 5). ROS 2 uses one DDS domain where all topics are available. Each module has to be part of this domain in order to subscribe to a topic and to automatically discover other systems. A router at the top level of the topology interconnects the subnets and acts as an internet gateway.

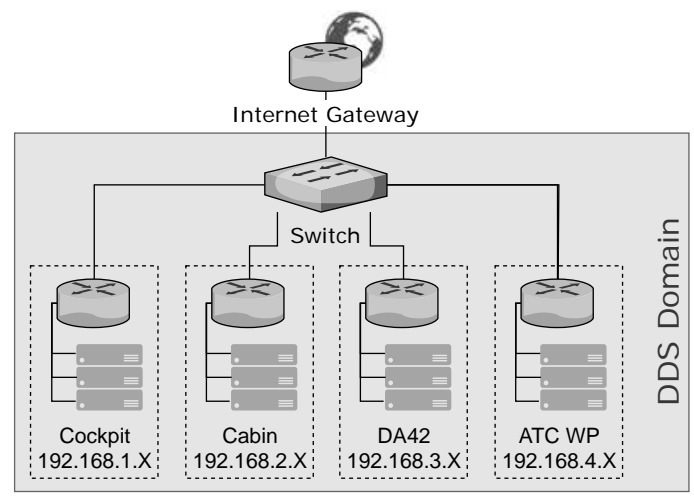

Figure 5: Envisaged network topology.

\section{Conclusion}

We presented our full-scale aircraft cabin section comprising the structure and electronic systems of a shortto medium-haul single-aisle commercial aircraft. The development process as well as the challenges and their solutions were illustrated on the basis of examples, particularly the implementation of an outside view simulation. The subsequent evaluation showed promising results concerning the level of immersion generated by the simulator. The next step will be the development of an interconnection infrastructure between the simulator entities.

\section{References}

[1] Richards LG. On the Psychology of Passenger Comfort. Human Factors in Transport Research. 1980;2:15 - 23.

[2] Vink P, Bazley C, Kamp I, Blok M. Possibilities to improve the aircraft interior comfort experience. Applied Ergonomics. 2012;43(2):354 - 359.

[3] Kwon G, Hong J, Lee T, Lee W, Park J, Tak T. Development of Real-Time Data Publish and Subscribe System Based on Fast RTPS for Image Data Transmission. In: Proceedings, 16th International Conference on Accelerator and Large Experimental Physics Control Systems (ICALEPCS 2017): Barcelona, Spain, October 8-13, 2017. 2018; pp. 473 - 477. 